\title{
Zusammenhänge überblicken, das Wissen auffrischen und den Nachwuchs für Rheumatologie begeistern
}

\section{Axiale Spondyloarthritis}

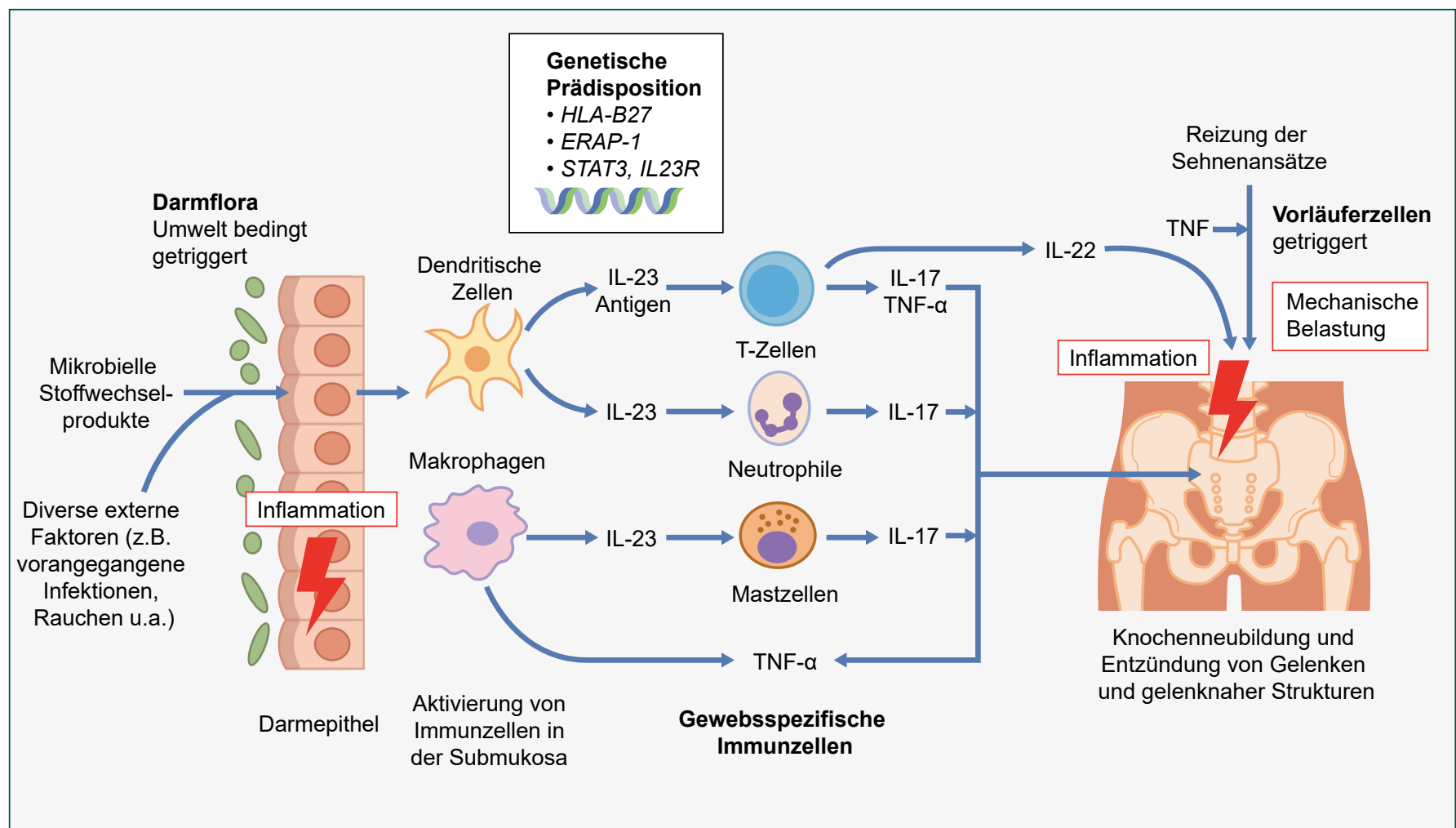

Aktuelles Verständnis der Pathogenese bei axialer Spondyloarthritis.

Autor:

Dr. Fabian Proft, Rheumatologie am Klinikum Benjamin Franklin -

Grafik kombiniert und modifiziert nach:

Brown MA et al. Nat Rev Rheumatol 2016;12:81-91.

Medizinische Klinik für Gastroenterologie, Infektiologie und

Loris RJ et al. Nat med $2012: 18: 1018-9$.

Ronneberger M. et al. Curr Rheumatol Rep 2011; 13:416-420.

Rheumatologie, Charité - Universitätsmedizin Berlin

Younis AA-R muhadharaty.com (2019) Spondyloarthropathie 
Leitlinien

\section{International standardisierte MS-Bildgebung ist notwendig für Prädiktionsmodelle}

Wie unterstützen Leitlinien Ärzte und Ärztinnen im klinischen Alltag, wie profitieren Patienten und Patientinnen von ihnen und wie sieht der Prozess beider Entstehung von Leitlinien aus, gerade wenn sie international ausgerichtet sind? Darüber hat Isabel Merchan Casado von der Deutschen Röntgengesellschaft (DRG) Professor Mike P. Wattjes vom Institut für Diagnostische und Interventionelle Neuroradiologie der Medizinischen Hochschule Hannover gesprochen. Wattjes ist verantwortlicher Erstautor der neuen internationalen Empfehlungen zur Bildgebung bei Multipler Sklerose (MS). Die Empfehlungen werden sowohl von den europäischen als auch von den nordamerikanischen MS-Organisationen unterstützt und sind in der renommierten Fachzeitschrift The Lancet Neurology veröffentlicht worden.

DRG: Multiple Sklerose (MS) ist eine chronisch entzündliche Erkrankung des zentralen Nervensystems. Hierzulande sind rund 250000 Menschen betroffen. Welche Rolle spielen die Radiologie und die Magnetresonanztomografie (MRT) bei der Diagnostik und Behandlung von MS?

Prof. Wattjes: Die Bildgebung durch die MRT bei der MS hat in den letzten Jahren stark an Bedeutung gewonnen. Früher war es so, dass die älteren Diagnosekriterien noch auf fast rein klinischen und laborchemischen Befunden beruhten. Mit der Verbesserung der MRT und besonders deren breiter Verfügbarkeit hat sie die Diagnose, aber auch die Verlaufsbeobachtung der MS quasi revolutioniert: Mit dem MRT kann man noch sensitiver vorgehen und Veränderungen im Gehirn und im Rückenmark darstellen, ohne dass der Patient klinische Symptome aufweist. Zum einen ermöglicht dies die Diagnose einer MS zu einem frühen Zeitpunkt, das heißt bei einem erstmaligen neurologischen Schub. Zum anderen ist dies besonders für die Verlaufsbeobachtung wichtig, denn man möchte ja in der Therapie keine neue Krankheitsaktivität mehr sehen. Die Möglichkeiten des MRT geben dem Neurologen Hinweise, etwa ob die Medikamente, die der Patient beziehungsweise die Patientin einnimmt, effizient genug sind, oder ob die Behandlung verstärkt und zu einer noch effektiveren Behandlung übergegangen werden muss.

DRG: Sie haben nun gemeinsam mit Experten und Expertinnen aus verschiedenen Ländern neue internationale Leitlinien zur Bildgebung bei MS in der klinischen Routinepraxis erarbeitet. Wie wichtig ist es, dass Ärzte und Ärztinnen auch international und nicht nur in Deutschland oder Europa bei dieser Krankheit nach einheitlichen Standards arbeiten?

Prof. Wattjes: Es ist sehr wichtig, dass diese Standardisierung der MS-Bildgebung auf internationalen Füßen steht, denn wesentliche Fragen, die wir jetzt und in der Zukunft für MS-Patienten beantworten müssen, sind abhängig von großen Datensätzen und zwar von Datensätzen, die standardisiert und damit vergleichbar sind, also wesentliche Prädiktionsmodelle hinsichtlich der individuellen Krankheits-progression erlauben. Prädiktionsmodelle kann man nur mit riesigen Datensätzen entwickeln. Das Gleiche

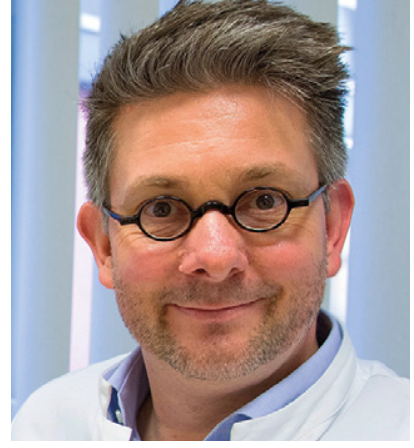

Prof. Mike P. Wattjes, Institut für Diagnostische und Interventionelle Neuroradiologie der Medizinischen Hochschule Hannover. (c) Karin KaiserMHH

gilt bei der Vorhersage von Behandlungserfolgen von einzelnen Medikamenten, die auch nur mit großen Datensätzen gelingen. Was den zukünftigen Einsatz automatisierter Bildauswertungsalgorithmen betrifft, sind wir auch von großen standardisierten Datensätzen abhängig. Diese sind nur möglich, wenn man sich medizinisch auf einen gewissen Grundstandard einigt - national und international.

DRG: Sind Sie bei der Entwicklung der Leitlinien zur MS Vorreiter oder haben Sie bereits vorhandene weiterentwickelt?

Prof. Wattjes: Diese 2021-Leitlinien, die wir nun geschrieben haben, beruhen auf einer Weiterentwicklung von Leitlinien, die im Jahre 2015 und 2016 von unterschiedlichen Gruppen bereits vorgestellt worden sind. Diese Leitlinien sind natürlich ständig im Fluss, es gibt immer neue Entwicklungen bei MS, neue Medikamente kommen auf den Markt, die kontroverse Diskussion über den Einsatz der Gadolinium-basierten Kontrastmittel et cetera. Insofern gab es mehrere Entwicklungen, die uns dazu veranlasst haben, die bestehenden Leitlinien zu revidieren und aufzufrischen. Dazu kommt, dass es uns ein großes Anliegen war, diese unterschiedlichen Leitlinien aus Europa und Nordamerika zu einer großen globalen Leitlinie zu homogenisieren.

DRG: Wie lange hat der Prozess der Leitbilderarbeitung bei Ihnen und der Gruppe von Experten und Expertinnen, mit der Sie zusammengearbeitet haben, gedauert?

Prof. Wattjes: Ich habe immer zu meiner Frau gesagt, es hat zwei Schwangerschaften gedauert, also 18 Monate. Es war eine sehr arbeitsintensive und aufregende Zeit und gegen Ende dieses Prozesses waren wir alle ein bisschen erschöpft. Am Ende des Tages hilft einem nur das Wissen um die Verantwortung gegenüber den Patienten über diesen anstrengenden und zeitraubenden Prozess hinweg. Aber Leitlinien sind wichtig - nicht primär für uns persönlich, sondern in erster Linie für die Patienten, für die Medizinentwicklung seitens der Pharmaindustrie sowie für Neurologen und (Neuro)Radiologen.

DRG: MS ist zwar therapier-, aber nicht heilbar. Betroffene müssen ein häufiges Monitoring und Verlaufskontrollen durchführen las- 
sen. Welche Empfehlungen geben Sie in den neuen Leitlinien etwa beim Thema Kontrastmittelgabe?

Prof. Wattjes: Dieses Thema war eine unserer Hauptmotivationen, um bestehende Leitlinien zu revidieren. Wir haben 2015 relativ unkritisch empfohlen, kontrastmittelverstärkte T1-gewichtete Sequenzen als MRT-Marker zu benutzen, um inflammatorische Krankheitsaktivitäten zu dokumentieren und damit auch zu untersuchen, ob eine medikamentöse Therapie effizient ist oder nicht. Doch wir haben nun die jüngste Diskussion um die Ablagerung von elementarem Gadolinium in der tiefen grauen Substanz zum Anlass genommen, diesen Aspekt zu revidieren. Wir sind jetzt der Meinung, dass man für viele Fragestellungen, insbesondere, was das Therapie-Monitoring betrifft, Kontrastmittel nicht mehr zwingend benötigt, weil wir eben mit anderen MRT-Parametern, mit den sogenannten aktiven T2-Läsionen, also mit neuen Entmarkungsherden, die auftreten, oder Entmarkungsherden, die 50\% größer werden im Vergleich zur Referenzuntersuchung, einen guten alternativen Marker haben, ohne auf Kontrastmittel zurückgreifen zu müssen. Selbstverständlich gibt es immer noch Indikationen, wo Kontrastmittel sinnvoll sind. Das gilt insbesondere für die Diagnose der MS. Aber für viele Indikationen, die die Verlaufsbeobachtung und das Therapie-Effizienz-Monitoring und das Sicherheitsmonitoring betreffen, sind Kontrastmittel nicht mehr zwingend notwendig und können eingespart werden.

DRG: Könnten Sie ganz generell beschreiben, wie die Diskussion und die Konsensfindung bei Leitlinien abläuft, auch international? Prof. Wattjes: Wichtig sind bei diesem Prozess mehrere Schritte, wobei die Vorbereitung der Konsensfindung das Entscheidende ist. Bei uns war es so, dass wir zunächst ein Konzept erarbeitet haben, in dem die Themen aufgeführt wurden, die einer möglichen Revision bedürfen. Dieses Proposal haben wir dann an die Leitlinienkommission verschickt, sodass sich jeder auf diese Konsensus-Findung vorbereiten konnte. Dann wurden die identifizierten Themen in einem KonsensusMeeting besprochen und soweit wie möglich evidenzbasiert diskutiert, Dieses Meeting hat 2019 in Graz stattgefunden. Entsprechend der Konsensus-Findung wurden alle diskutierten Aspekte dokumentiert. Nach dem zweitägigen Konsensus-Meeting wurden die Ergebnisse in ein Manuskript eingearbeitet. Danach zirkulierte dieses Manuskript unter den beteiligten Mitgliedern der Leitlinienkommission. In dieser etwas längeren Zirkulations- und Manuskriptphase wurden auch noch weitere neue Aspekte, die erst nach dem Konsensus-Meeting relevant wurden, in das Manuskript eingearbeitet.

DRG: Wenn man bedenkt, wie lange es dauern kann, bis Leitlinien erstellt sind und wie rasant sich die Medizin in manchen Bereichen entwickelt, könnte sich die Frage der Aktualität stellen. Wie schätzen Sie dieses Thema ein?

Prof. Wattjes: Zu lange darf ein solcher Prozess natürlich nicht dauern, weil dann neue Aspekte und Themen relevant werden, welche dann wieder berücksichtigt und eventuell eingearbeitet werden müssen. In unserem Fall war das nicht dramatisch, denn Innovationen auf dem Gebiet der Bildgebung bei der MS passieren nicht von einem Tag auf den anderen. Bis neue bildgebende Methoden so validiert sind, dass sie letztendlich auch Eingang in die klinische Praxis finden können, dauert es zum Teil mehrere Jahre. Bei anderen Leitlinien kann das aber anders aussehen. Insbesondere bei Therapieleitlinien kann beispielsweise die Zulassung eines neuen Medikaments die Therapielandschaft komplett verändern, sodass die entsprechenden Leitlinien umfänglich aktualisiert und angepasst werden müssen. Aber das war bei uns wie gesagt nicht der Fall.

DRG: Kritiker und Kritikerinnen monieren zuweilen, dass Leitlinien, auch wenn sie evidenzbasiert sind, von unzureichender methodischer Qualität sein können. Sie sehen darin die Gefahr, dass die in Leitlinien enthaltenen Empfehlungen bei ihrer Umsetzung nicht den gewünschten Effekt einer besseren Versorgung von Patienten und Patientinnen erreichen. Wie schätzen Sie diese Kritik ein?

Prof. Wattjes: Diese Kritik ist zum Teil berechtigt. Man kann Leitlinien häufig nicht ausschließlich evidenzbasiert ausrichten, weil es für viele Aspekte nicht genug Evidenz, zum Beispiel keine Klasse1-Evidenz gibt, um sie darauf begründen zu können. Viele Aspekte von Leitlinien, und das schließt auch unsere Empfehlung mit ein, beruhen häufig auf langjährigen Erfahrungen in der klinischen Routine. Dies wird zum Teil kontrovers diskutiert. Kritik an bestehenden Leitlinien sehe ich persönlich nicht negativ, denn in dem Moment, wo es Kritik an Leitlinien gibt, entsteht auch ein Dialog. Einen kritischen Dialog erfahre oder interpretiere ich als fruchtbar und motivierend, um die Dinge noch weiter zu verbessern. Leitlinien sind grundsätzlich nicht der Goldstandard, sondern sie sollen Mediziner leiten und dabei unterstützen, in der klinischen Routine die richtigen Entscheidungen zu treffen. Sie sind aber nicht verpflichtend. Es gibt klinische Situationen in denen man sich begründet über eine Leitlinie hinwegsetzen kann.

\section{DRG: In welchen medizinischen Fachgebieten sehen Sie aktuell} einen besonderen Nutzen oder eine besondere Notwendigkeit von Leitlinien?

Prof. Wattjes: Das sind Themen oder Fachgebiete, bei denen die zur Verfügung stehenden Daten für die Kollegen häufig nicht konklusiv und sehr schwierig zu interpretieren sind. Das gilt sowohl für radiologische beziehungsweise diagnostische Themen als auch für die Therapie von bestimmten Erkrankungen. Ein gutes Beispiel ist die aktuelle und kürzlich veröffentlichte DGN-Leitlinie für MS. Die MS-Therapielandschaft wird immer breiter und heterogener, und auch da ist es deshalb wichtig, den Ärzten mithilfe von Leitlinien Orientierung und Hilfestellung zu bieten, um so der bestmöglichen Therapieentscheidung den Weg zu bereiten, zum Wohle des individuellen Patienten.

\section{Literatur}

1. Hemmer B, et al.: Diagnose und Therapie der Multiplen Sklerose, Neuromyelitisoptica-Spektrum-Erkrankungen und MOG-IgG-assoziierten Erkrankungen, S2kLeitlinie, 2021, in: Deutsche Gesellschaft für Neurologie (Hrsg.), Leitlinien für Diagnostik und Therapie in der Neurologie. www.dgn.org/leitlinien

2. Wattjes MP, Ciccarelli O, Reich DS, et al.: 2021 MAGNIMS-CMSC-NAIMS consensus recommendations on the use of MRI in patients with multiple sclerosis. Lancet Neurol. 2021;S1474-4422(21)00095-8.

www.drg.de

DEUTSCHE RÖNTGENGESELLSCHAFT Gesellschaft tür medizinische Radiologie e.
128

Kompass Autoimmun 2021;3:126-129 DOI: $10.1159 / 000518486$ 


\section{Kampagne will die kommende Generation begeistern}

Das Bündnis für Rheumatologie hat die Nachwuchs-Kampagne rheuma2025.de an den Start gebracht. Das Kernziel ist, bis zum Jahr 2030 rund 800 Ärzte und Ärztinnen rheumatologisch weiterzubilden, um die Versorgung der Patientinnen und Patienten auch zukünftig sicherzustellen. Partner des Bündnisses sind die Deutsche Gesellschaft für Rheumatologie, neben dem Berufsverband Deutscher Rheumatologen und dem Verband Rheumatologischer Akutkliniken, mit Unterstützung der RheumaAkademie. Die Kampagne setzt das Bündnis mit der Designagentur Lindgrün aus Berlin um.

Haupt-Adressat der Kampagne ist der rheumatologische Nachwuchs. So soll der Kampagnen-Hashtag \#rhmtlgy vor allem Studierende und angehende Rheumatologen ansprechen. Für den Zugang zu den Zielgruppen wird die Kampagne verschiedene Kanäle nutzen: die Website, Instagram, Twitter, Videos, Podcasts, Chats und Gespräche - bis hin zu Treffen und einer geplanten Roadshow vor Ort an den Universitäten.

Pandemiebedingt läuft die Kampagne derzeit virtuell an. Die Website rheuma2025.de bildet den Auftakt: Sie bietet Informationen über Rheumatologie, Tipps und Lernkarten, bundesweite Kontakte zu rheumatologischen Ansprechpartnern und Terminhinweise. Des Weiteren verlinkt sie zu den Social-Media-Kanälen. Es gibt beispielsweise den Facebook-Account R_rhmtlgy und den gleichnamigen YouTube-Kanal.

Auf Twitter ist der Journal Club «JC.rhmtlgy» geplant. Hier diskutieren Wissenschaftler aktuelle Fachartikel und Forschungsthemen. Im Videoformat «FreiGang» will der Medizinstudent Jonathan Beron
Persönlichkeiten aus der Rheumatologie interviewen. Zudem soll ein Kampagnen-Quiz geben. Prof. Heinz-Jürgen Lakomek, Geschäftsführer des Verbandes Rheumatologischer Akutkliniken, sagt: «Auch in diese Quiz-Fragen fließt gebündeltes rheumatologisches Wissen ein. Unsere Expertinnen und Experten stellen authentische Fälle vor und entwickeln dazu passende Fragen». Alle Lernkarten basieren demnach auf dem Skriptum Rheumatologie der Deutschen Gesellschaft für Rheumatologie. Zudem orientieren sich die Fragen an den studentischen Prüfungsfragen des Instituts für medizinische und pharmazeutische Prüfungsfragen. Dr. Silke Zinke, Vorsitzende des Berufsverbandes Deutscher Rheumatologen, erläutert: «Rheumatologen müssen neben sehr umfassenden internistischem Wissen auch geradezu detektivisches Geschick entwickeln, um den systemischen Krankheiten auf die Spur zu kommen. Diesen Rätselfaktor sprechen wir in der Kampagne mit einer Geheimschrift an, die die Designagentur Lindgrün eigens dafür entwickelt hat.»

Den Erfolg des Projekts will das Bündnis messen. Im Auftrag der Deutschen Gesellschaft für Rheumatologie untersuchen Gesundheitswissenschaftler der Universität Erfurt in mehreren Wellenbefragungen den Wissensstand der Studierenden zum Start und nach Ende der Kampagne.

www.rheuma2025.de

Im Interview

\section{Prof. Hanns-Martin Lorenz}

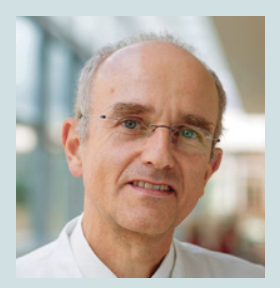

Prof. Dr. Hanns-Martin Lorenz, Universitätsklinikum Heidelberg, Leiter Sektion Rheumatologie/Medizinische Fakultät und Mitglied im Beirat der DGRh
Kompass Autoimmun (KAl): Herr Professor Lorenz, wie viele Ärzte absolvieren jedes Jahr die Weiterbildung für Rheumatologie? Prof. Lorenz: Genau wissen wir das leider gar nicht, aber in etwa 50 Ärztinnen und Ärzte legen jährlich die Facharztprüfung ab. Bei einer Weiterbildungsdauer von 6 Jahren in der Rheumatologie macht das etwa 300 fertige Rheumatologen in 6 Jahren. Das ist für die Versorgung der Bevölkerung schon heute, aber erst recht in $\mathrm{Zu}$ kunft, viel zu wenig.

\section{KAl: Genau hierfür ist ja die Kampagne gedacht:}

Sie wollen angehende Ärztinnen und Ärzte für das Fach Rheumatologie begeistern.

Prof. Lorenz: Aber damit ist es nicht getan! Wir müssen die Anzahl der Weiterbildungsstellen für die Bewerberinnen und Bewerber erhöhen, hier ist der eigentliche Flaschenhals. Die Politik muss endlich auf den Versorgungsbedarf der Bevölkerung mit rheumatologischen
Fachärzten reagieren. Um das zu bessern, bräuchten wir schon heute in jedem größeren Zentralkrankhaus einen Weiterbildungsberechtigten für Rheumatologie. Leider fördert das pauschalierte Abrechnungssystem der diagnosebezogenen Fallgruppen (DRG-System) eine Kultur hin zu DRG-reichen und damit lukrativen Fächern in den Krankenhäusern. Die sprechende Medizin, zu der u.a. auch die Rheumatologie zählt, fällt somit in den Krankenhäusern hinten runter, entsprechend gibt es keine ausreichenden Weiterbildungsstellen.

\section{KAl: Was schlagen Sie vor?}

Prof. Lorenz: Die Politik muss die Krankenhausfinanzierung von der Finanzierung der Weiterbildung trennen. Nur so lässt sich dem Flaschenhals der Weiterbildungsstellen gegensteuern. Andere europäische Länder machen uns das erfolgreich vor. Hierzulande hat die Einführung der ambulant-spezialärztlichen Versorgung Rheumatologie an den Spezialkliniken etwas gebessert, das genügt in der Fläche aber bei Weitem nicht, um so viele RheumatologInnen weiterzubilden wie wir bräuchten

KAl: Herr Professor Lorenz, vielen Dank für dieses Gespräch. 